\title{
Clase Inversa online o/y presencial, para mejorar los resultados académicos
}

\section{Amparo Ribes-Greus a}

aDepartamento de Máquinas y Motores Térmicos. Grupo GIIMA. Escola Tècnica Superior de Enginyeria Industrial ETSEI, Universitat Politècnica de València UPV. aribes@ter.upv.es

\section{\$ EWWDW}

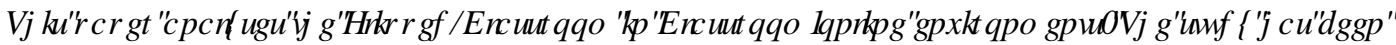

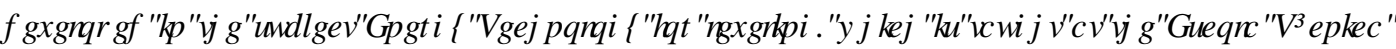

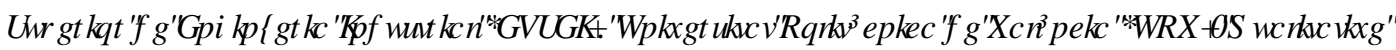

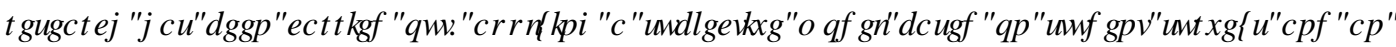

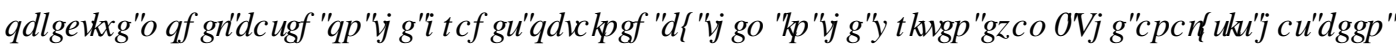

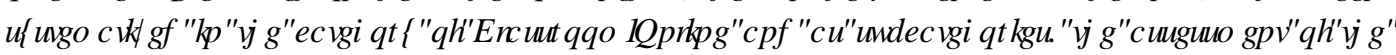

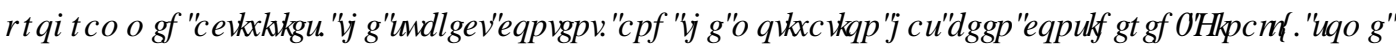

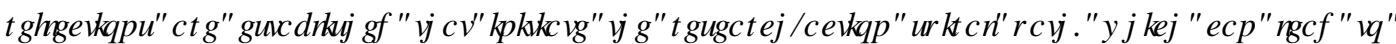

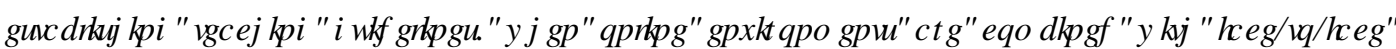
HQYILRQP HQWD

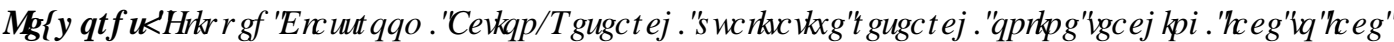

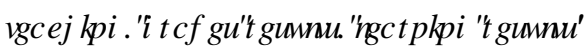

\section{HXP HQ}

En este trabajo se analiza la implementación de la Clase Inversa en entornos presenciales y online. El estudio se ha llevado a cabo en la asignatura es Tecnología Energética para nivelación, que se imparte en Escola Tècnica Superior de Enginyeria Industrial (ETSEI), Universitat Politècnica de València (UPV). Se ha realizado una investigación cualitativa, aplicando tanto, un modelo subjetivo basado en las encuestas de los alumnos, como un modelo objetivo, fundamentado en las notas obtenidas por éstos en el examen escrito. El análisis se ha sistematizado en la categoría de Clase Presencial/Clase online y como subcategorías se ha considerado la valoración de las actividades programadas, la relación de contenidos y la motivación. Finalmente se establecen algunas reflexiones que inician el camino espiral Investigación-Acción, para establecer pautas docentes cuando se intercalen los entornos online con los entornos presenciales.

Palabras clave: Clase Inversa, investigación-Acción, investigación cualitativa, docencia online, docencia presencial, resultados académicos, resultados de aprendizaje.

\section{Introducción}

La pandemia, causada por el Covid-19, ha afectado de manera especial a la actividad docente universitaria, de modo que, a lo largo de este curso, se ha producido una presencialidad cambiante, según las circunstancias sanitarias, combinada con la necesaria virtualidad síncrona o asíncrona. Es evidente que, en estos momentos de excepcionalidad, se ha producido un proceso masivo de adaptación inmediato, y sin planificación previa, a la docencia online. En muchos foros, por las características circunstanciales de esta docencia, se le ha llamado docencia no presencial de emergencia, puesto que se ha basado en el aprendizaje, por parte de los docentes y lo más rápido posible, del uso de las TIC (Tecnologías de la Información y la 
Comunicación), para impartir la docencia asignada. En muchas ocasiones, la docencia online se ha supeditado al uso de dichas TIC. Hay que tener en cuenta que ambas se apoyan mutuamente, pero no es lo mismo, ya que las TIC necesitan de una metodología adecuada que las contextualice y les de valor.

No obstante, dado que todos los docentes se han visto obligados al cambio, por la propia imposición que ha forzado la pandemia, hay una parte significativa de ellos que está descubriendo el potencial y la enorme capacidad de los entornos online en el proceso de enseñanza-aprendizaje, y apuestan por una profunda reflexión sobre el nuevo paradigma educativo, como pedagogía innovadora en un mundo interconectado, más allá del mero uso de las aplicaciones informáticas. Se trata de aprovechar la capacidad que ofrecen los entornos online para el trabajo cooperativo, el intercambio activo de información, etc. En este caso, el docente es el que piensa, diseña y propone espacios, donde la prioridad es el aprendizaje y donde los alumnos aprenden.

En este trabajo, se apuesta por reflexionar sobre los efectos de la docencia presencial/ docencia online, en los resultados académicos y consecuentemente de aprendizaje en una asignatura concreta, en la que se han aplicado metodologías activas y la Clase inversa presencialmente, durante los cursos 2018-2019, 20192020 y online durante el curso 2020-2021. La asignatura es Tecnología Energética para nivelación y se imparte en Escola Tècnica Superior de Enginyeria Industrial (ETSEI), Universitat Politècnica de València (UPV). Se trata de una asignatura de carácter optativo en la titulación 2242 del master universitario en ingeniería industrial (acceso desde grado de mecánica o de ingeniería química) en el módulo I de complementos de Tecnologías Industriales con 4,5 créditos distribuidos en 2,4 créditos de teoría y 2,1 créditos de prácticas. En esta asignatura se aborda el estudio de las principales fuentes de energía, así como las transformaciones energéticas más importantes, evaluando la eficiencia de las mismas. La asignatura se desarrolla mediante la aplicación de diferentes técnicas docentes que se van alternando a lo largo del curso, en función de los objetivos y resultados de aprendizaje concretos que se pretenden conseguir.

El núcleo fundamental de la metodología aplicada en esta asignatura es la Clase Inversa o Flipped Classroom, combinada con un modelo de aprendizaje Cooperativo basado en la resolución de problemas y proyectos reales. La clase inversa no responde exactamente a un modelo de clase semipresencial, porque no contempla una reducción de los tiempos de clase presencial, sino que se hace un uso distinto del tiempo, LLAMAZARES ( 2015). La idea de la Clase Inversa es modificar el orden metodológico y dedidar el tiempo presencial a orientar y guiar a los estudiantes, en lugar de impartir clases magistrales, MERLA (2016). Diferentes estudios realizados en el ámbito universitario demuestran que la metodología de clase inversa incide positivamente en el alumnado, SALAS- RUEDA, (2019), SOSA, (2018), MORALES (2019) y es muy eficaz en el proceso de aprendizaje, ya que facilitar el desarrollo de habilidades cognitivas de orden superior, puesto que estas requieren un mayor esfuerzo por parte de los estudiantes y se pueden alcanzar de forma cooperativa o individualizada con la ayuda del docente, LAG, (2019), FOLDNES, (2016).

Para obtener resultados significativos se ha elegido la metodología de Investigación - Acción, ELLIOTT, (1993), ya que supone un proceso de continua búsqueda, conlleva integrar la reflexión sistemática sobre la práctica y estimula a introducir mejoras progresivas. Además, se ha elegido la Clase Inversa como práctica docente de comparación porque la presencialidad del profesor como agente motivador y director del proceso de aprendizaje, es el eje de esta metodología, CASTILLA (2017). El profesor tiene que pensar la asignatura en términos de competencias, clasificar los resultados en función de su complejidad, planificar las tareas, elegir las actividades, buscar casos reales adecuados, poner en contacto a los estudiantes, formar grupos de debate, resolver dudas, realizar evaluaciones formativas y detectar posibles fallos del proceso de aprendizaje, etc., pero estas tareas son comunes a las que realiza el docente en el entorno online. En realidad, son tareas comunes en ambos entornos de aprendizaje, y por lo tanto, se puede tomar como punto de partida 
la Clase Inversa para comprobar si los resultados de aprendizaje son similares en ambos entornos docentes y reflexionar sobre la presencia del docente en contextos de enseñanza-aprendizaje presencial/online.

Este estudio se basa en un caso particular muy concreto, la asignatura de Tecnología energética para nivelación, por lo tanto, se trata de una investigación docente limitada, porque la población a la que se aplica se reduce al entorno del aula. No obstante, el desarrollo de esta investigación es exportable a otras asignaturas, de modo que se puede generar una base de conocimiento que establezca pautas para comprender el contexto multidimensional online/presencial, donde la comunicación entre los protagonistas (docentes y estudiantes) es más compleja y requere de habilidades específicas en cada uno de los entornos. Las variables espacio-tiempo se viven de forma peculiar en un ámbito online o presencial, como se ha comprobado en este último año. En el entorno online no existe el límite en horas de dedicación y se evidencian valores muy distintos, por lo que es necesario comparar los resultados finales en ambos procesos pedagógicos.

\section{2.- Objetivos}

El objetivo principal es comparar la implementación de la Clase Inversa en entornos de enseñanzaaprendizaje presencial y online, para establecer pautas que optimicen el proceso. Para ello, los objetivos parciales que se plantean son:

- Contrastar la programación y actividades en las distintas etapas del proceso de aprendizaje desarrolladas en la Clase Inversa online o presencial

- Interpretar los resultados de aprendizaje alcanzados por los estudiantes en la Clase Inversa online o presencial

- Revisar el papel del docente en el entorno presencial y online.

\section{3.- Desarrollo de la innovación}

La innovación llevada a cabo en este trabajo se basa en la implementación de la Clase Inversa en un entorno online o presencial. En la Tabla 1 se muestra cómo se ha puesto en práctica y se evalúan las estrategias de acción llevadas a cabo. Se han esquematizado las tareas asociadas a cada objetivo y los indicadores de medida para valorar la efectividad de dichas tareas.

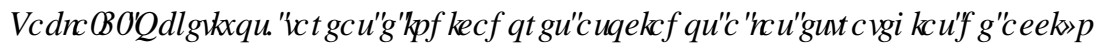

\begin{tabular}{|l|l|l|}
\hline OBJETIVOS & TAREAS & INDICADORES \\
\hline OBJ 1 & Identificar los resultados de & Guía Docente \\
Contrastar la & aprendizaje & Encuestas realizadas por \\
programación y & Seleccionar/crear contenidos & responsables de clase \\
actividades en las & Diseñar actividades de aprendizaje & inversa etc. \\
distintas etapas del & Establecer el sistema de evaluación & Encuesta realizada \\
proceso de aprendizaje & Plasmar todo en una planificación & después de llevar a cabo \\
desarrolladas en la & completa, semana a semana que se & la actividad de Elevator \\
Clase Inversa online y & publicará en Lessos y contenidos de \\
presencial & poliformaT & Pitch \\
\hline
\end{tabular}

(c) EY-NC-ND 2021, Universitat Politècnica de València

CRQJUMR, Q5 HGHपिए। 


\begin{tabular}{|l|l|l|}
\hline & $\begin{array}{l}\text { Formación de grupos mediante la } \\
\text { técnica de elevator PITCH } \\
\text { Estas tareas son comunes en la } \\
\text { Clase inversa online y presencial }\end{array}$ & \\
\hline $\begin{array}{l}\text { OBJ 2 } \\
\text { Interpretar los } \\
\text { resultados de } \\
\text { aprendizaje alcanzados } \\
\text { por los estudiantes en } \\
\text { la Clase inversa online } \\
\text { y presencial }\end{array}$ & $\begin{array}{l}\text { Preparación de pruebas objetivas en } \\
\text { un examen escrito presencial } \\
\text { Análisis de los resultados obtenidos }\end{array}$ & $\begin{array}{l}\text { Estudios estadísticos de } \\
\text { los resultados obtenidos } \\
\text { en los cursos 2016-2017, } \\
2018-2019,2019-2020\end{array}$ \\
\hline $\begin{array}{l}\text { OBJ 3 } \\
\text { Revisar el papel del } \\
\text { docente en el entorno } \\
\text { presencial y online }\end{array}$ & formulación de preguntas & $\begin{array}{l}\text { Iniciar la espiral de } \\
\text { mejora que supone la } \\
\text { investigación-acción }\end{array}$ \\
\hline
\end{tabular}

El desarrollo de la asignatura, tanto online como presencial esta recogido en una guía docente que se ha titulado Cuaderno Guía, en el que se indica semana a semana las actividades que se van a realizar.

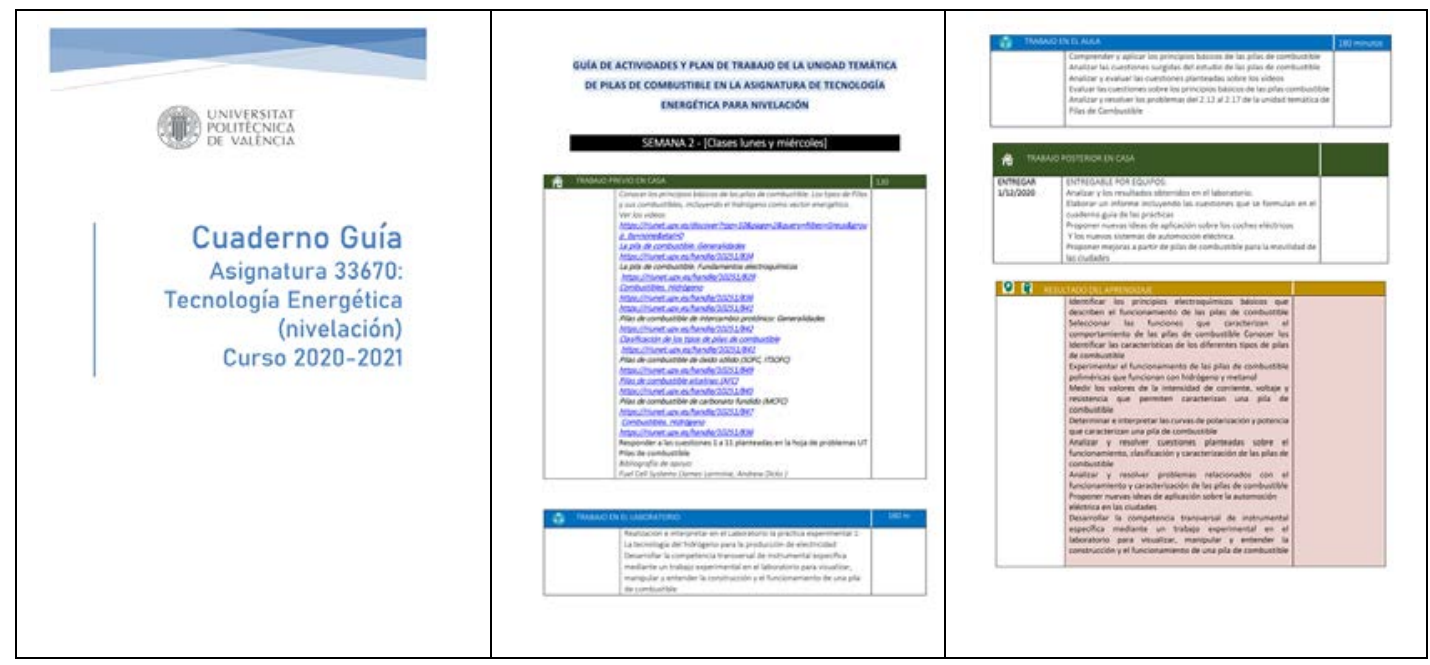

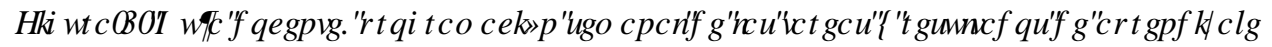

Además, en el proceso de aprendizaje se recurre al trabajo cooperativo, para fomentar el aprendizaje entre iguales. En ese sentido, la formación de los equipos es una acción importante y se aplicará la técnica de innovación Elevator Pitch. 


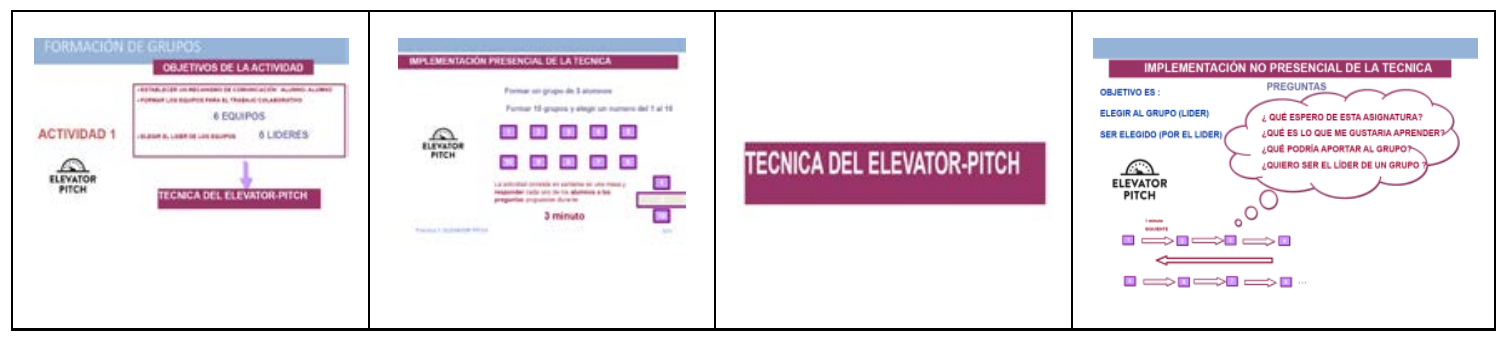

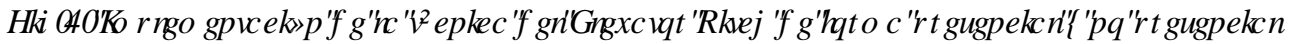

Esta técnica nace de la necesidad de vender nuestra oferta en cualquier momento, a cualquier persona susceptible de comprar, y en el menor tiempo posible. Los alumnos deberán preparar su marca personal con el objetivo de formar los equipos. Tenerla preparada es importante, porque en algunas entrevistas de trabajo ya se usan estas técnicas. Con ello se crearán los equipos de clase, que se mantendrán en el transcurso de la asignatura.

Esta tarea no puede ser la misma en un entorno online que presencial. La actividad online se realiza de forma individual. En la actividad presencial los alumnos se presentan en un grupo que va cambiando a lo largo de la sesión hasta que todos se conocen. En la Figura 2 se muestra la implementación de la técnica del Elevator Pitch de forma presencial y no presencial.

La evaluación es una parte importante en el proceso de aprendizaje. En este caso, se evalúan todas las actividades realizadas por los alumnos. Las tareas evaluables son los entregables (10\%); las prácticas de laboratorio (15\%); el trabajo de innovación que consiste en la mejora energética de un proceso industrial $(25 \%)$ y el examen escrito individual (50\%).

\section{4.- Resultados}

El estudio se ha realizado en una población reducida, ya que el número de alumnos que cursan la asignatura oscila entre 35-60, pero se ha aplicado a tres cursos consecutivos. En él se tienen en cuenta las opiniones de los estudiantes, mediante encuestas que se realizan después de alguna actividad y los resultados académicos que siempre están relacionados con los resultados de aprendizaje o capacidades cognitivas desarrolladas.

La investigación que se plantea para analizar los resultados es cualitativa, está orientada a la comprensión y tal vez, al cambio o a la toma de decisiones CASTILLO, (2003). En el desarrollo de esta investigación se han cumplido las cuatro fases que constituyen Investigación- Acción ELLIOTT, (1993):

1. Diagnosticar una situación para la práctica, en este caso comparar la clase presencial y la clase online

2. Formular estrategia de acción que en este caso concreto sería la implementación de la Clase Inversa

3. Poner en práctica y evaluar las estrategias de acción

4. Reflexionar el resultado para establecer un diagnóstico de la situación, iniciándose así la consiguiente espiral de reflexión y acción

Los resultados que se presentan en este trabajo responden a diferentes modelos de análisis, ya que se combina el PRGHRIXENAMVR y HQP RGHARRENAMAR. El primer modelo proporciona la información a partir de las opiniones de los estudiantes, en cierto modo subjetivas, vinculada a actitudes y por lo tanto expuestas 
a captar la singularidad de las situaciones particulares. Como datos para aplicar el P RGHRIFHQWIFDP HQWH $R E N A M A R$ se ha elegido las notas de los estudiantes durante los cursos que se analizan en este trabajo.

Para alcanzar el objetivo 1 se ha aplicado el modelo subjetivo, en la Tabla 2 se recogen las opiniones de los estudiantes respecto a la implementación de la Clase Inversa online durante el curso 2020-2021 (han contestado 10 estudiantes de 36), y de la Clase Inversa presencial durante el curso 2019- 2020 (han contestado 8 estudiantes de 48). No se disponen de encuestas en los otros cursos. Las preguntas elegidas están relacionadas con las actividades programada, la relación de contenidos y la motivación.

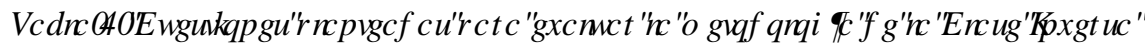

\begin{tabular}{|l|c|c|}
\hline \multicolumn{1}{|c|}{ Cuestiones planteadas } & $\mathbf{2 0 2 0 - 2 0 2 1}$ & $\mathbf{2 0 1 9 - 2 0 2 0}$ \\
Online & $\mathbf{p}$ & $\begin{array}{c}\text { prencial } \\
\text { \% }\end{array}$ \\
\hline No ha ido a las sesiones de clase con un trabajo previo realizado & 60 & 50 \\
\hline Se ha fomentado el trabajo en grupo en el aula/Teams & 50 & 100 \\
\hline He llegado mejor preparado a los exámenes & 20 & 50 \\
\hline $\begin{array}{l}\text { Las actividades propuestas en las sesiones ayudaban a profundizar en } \\
\text { el aprendizaje }\end{array}$ & 40 & 50 \\
\hline $\begin{array}{l}\text { Mantendría el repaso y resolución de dudas de las actividades previas a } \\
\text { la sesión }\end{array}$ & 60 & 37,5 \\
\hline Mantendría la explicación de nuevos contenidos teóricos & 60 & 50 \\
\hline Mantendría las exposiciones y presentaciones de los alumnos & 60 & 50 \\
\hline $\begin{array}{l}\text { Mi aprendizaje ha sido más activo y experiencial, lo cual ha aumentado } \\
\text { mi compromiso con la asignatura }\end{array}$ & 40 & 67,5 \\
\hline \begin{tabular}{l} 
Mantendría la explicación de nuevos contenidos teóricos \\
\hline
\end{tabular} & 60 & 50 \\
\hline Estaría de acuerdo en realizar actividades de gamificación & 10 & 25 \\
\hline
\end{tabular}

La actividad de Elevator Pitch se ha evaluado siguiendo el modelo subjetivo, en la Tabla 3 se muestran las opiniones de los estudiantes, la encuesta se ha realizado en Socrative durante el curso 2020-2021 y (han contestado 29 estudiantes), y durante el curso 2019- 2020 (han contestado 37 estudiantes).

Con referencia al modelo subjetivo es posible pensar que las conclusiones que se obtengan de un número de respuestas tan reducido, frente al número total de alumnos, son infundadas o inexactas, porque el número de las opiniones no responde a todos los alumnos y no se dispone de las encuestas de todos los cursos académicos. No obstante, es un punto de partida, incluso una oportunidad, para analizar el modo en que se desarrolla la docencia y aprender acerca de la propia actividad. (CASTILLO (2003). 


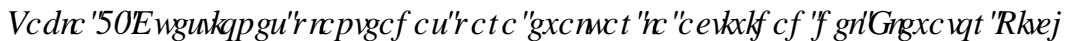

\begin{tabular}{|l|c|c|}
\hline \multicolumn{1}{|c|}{ Cuestiones planteadas } & $\begin{array}{c}\mathbf{2 0 2 0 - 2 0 2 1} \\
\text { Online } \\
\mathbf{\%}\end{array}$ & $\begin{array}{c}\mathbf{2 0 1 9 - 2 0 2 0} \\
\text { presencial } \\
\mathbf{\%}\end{array}$ \\
\hline La actividad es adecuada para formar los grupos & 86,36 & 100 \\
\hline $\begin{array}{l}\text { Tal cómo se plantea la actividad, pensar en mi marca personal } \\
\text { puede ayudarme en una entrevista de trabajo }\end{array}$ & 95,45 & 100 \\
\hline He encontrado la actividad útil para mí formación & 77,27 & 89,03 \\
\hline Si volviera a hacer esta actividad, me la prepararía mejor & 63.64 & 45,33 \\
\hline
\end{tabular}

Para sistematizar el análisis subjetivo, se han establecido diferentes categorías para organizar la reflexión. La primera categoría estaría relacionada con la presencialidad/ no presencialidad de la docencia y las subcategorías serían la valoración de las actividades programadas, la relación de contenidos y la motivación.

De las respuestas de los alumnos se puede deducir:

- Durante la clase presencial el profesor mejora la percepción que tienen los estudiantes sobre las actividades realizadas en el aula, la preparación del examen, el compromiso por la asignatura etc.

- La percepción sobre el contenido de la asignatura y la realización de las tareas es similar, tanto en un entorno presencial, como en un entorno online. Sin embargo, en la clase online cobra más relevancia el papel de las tutorías.

- Por la experiencia acumulada, y por los resultados de las encuestas, parece que (D)PRWDFFy IQNQMFDbasada en actividades de gamificación y de desarrollo del pensamiento lateral no están bien aceptadas, sólo algunos estudiantes aprecian su potencial.

Para alcanzar el objetivo 2 se aplica el PRGHRIFIHQWIFDP HQUARENAMNRПAsí, se analizan las notas de los estudiantes durante los cursos señalados. En figura 3 se representa las estadísticas de las notas obtenidas por los estudiantes en los tres cursos elegidos para este estudio. Se ha añadido un año anterior a la aplicación de la Clase Inversa (2016-2017), para comparar las mejoras. La nota media de los alumnos aprobados se situa alrededor de 5.5 y 6.5, durante la Clase inversa con docencia presencial (curso 2018-2019, 2019-2020, respectivamente), mientras que la nota media con la docencia online está alrededor de 7,5. Sin embargo, el número de alumnos suspendidos con notas inferiores a 3,5 es mucho más alto durante la docencia online que en todos los otros cursos estudiados. Es decir, existe una tendencia creciente a la polarización de los resultados y la nota de los alumnos aprobados es mucho más alta cuando la docencia es online.

Asimismo, resulta interesante comprobar cómo evoluciona, a lo largo de los diferentes cursos, el número de alumnos que obtienen los mejores resultados, los peores y los intermedios en la franja de aprobados y suspendidos. En la Figura 4 se han representado los porcentajes de los cuatro bloques en los que se han agrupado las notas. Se puede comprobar que en la Clase Inversa online el porcentaje de alumnos con las notas más altas ha aumentado significativamente. 

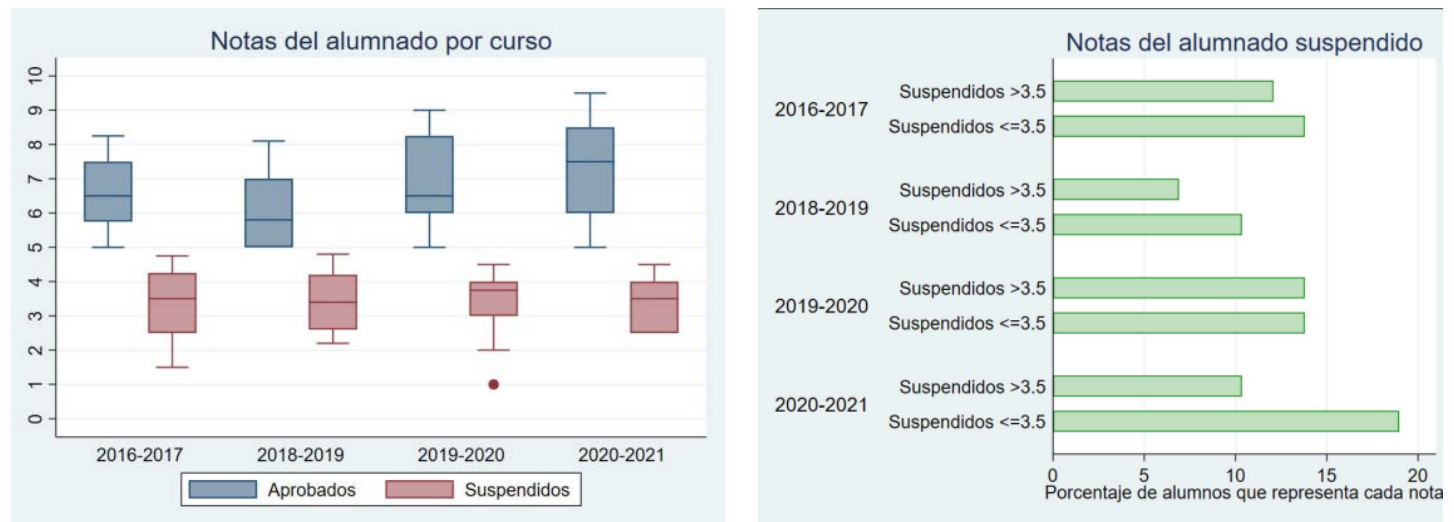

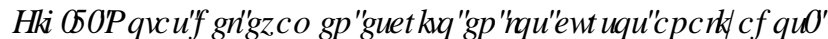

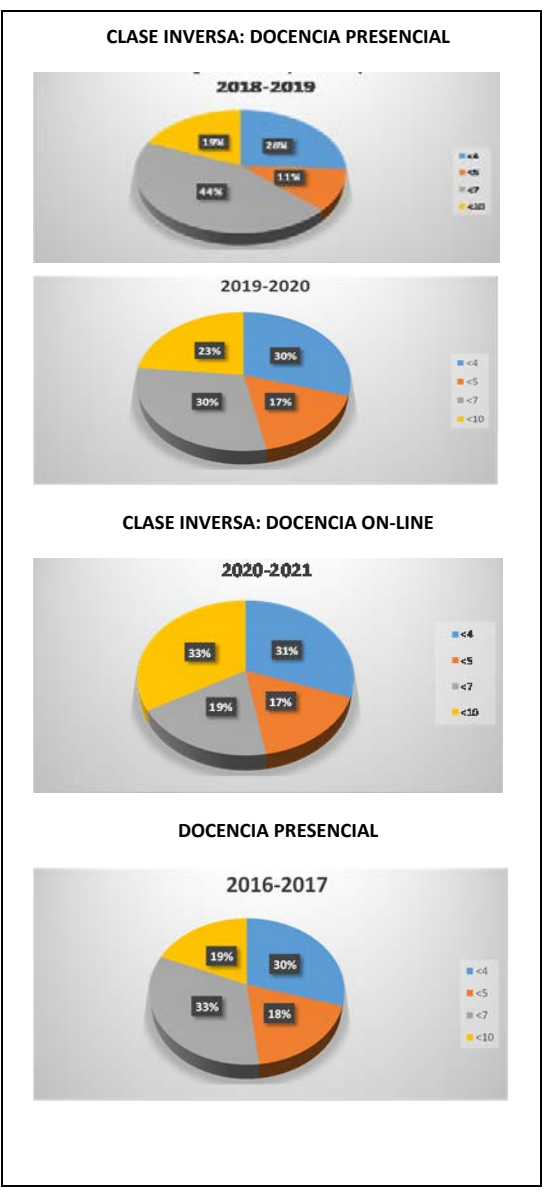

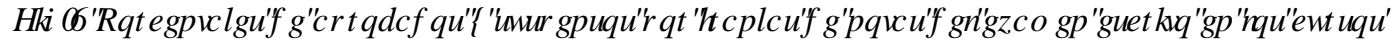 $D Q D D] D O R V I$}

Este hecho se puede interpretar considerando que se produce una clara separación entre los alumnos que se motivan, se conectan todos los días a la clase online y siguen sistemáticamente la asignatura y los que no se conectan y la abandonan. Sin embargo, la Clase inversa ofrecer a los estudiantes la oportunidad de mantener el contacto con la asignatura y re-engancharse a ella si así lo deciden. 
Por último, para alcanzar el objetivo 3, se realiza una reflexión crítica del proceso en los dos ámbitos presencial/online, para clarificar cada situación, lo que puede llevar en un futuro a tomar las decisiones pertinentes. En base a los datos obtenidos se pueden formular preguntas abiertas para clarificar cada situación. ¿Por qué las actividades que se proponen no consiguen el efecto deseado? ¿Cuáles son las causas por las que, en el entorno online, los alumnos perciben un menor compromiso por la asignatura? $¿$ Tal vez influye la posibilidad de desconectarse en cualquier momento, mientras que en un entorno presencial no es habitual que un alumno salga a mitad de la clase? ¿Hay posibilidades de revertir esta situación? ¿Qué se puede mejorar? ¿Cómo se tiene que fomentar la participación del alumno en el entorno online?

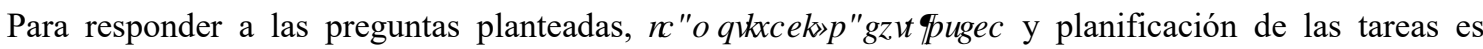
fundamental. El aprendizaje mediante proyectos basados en experiencias reales, detectando problemas y buscando soluciones, parece que es la forma correcta de proceder. Un proyecto de innovación relacionado con las necesidades de nuestro entorno cercano o mundial, (por ejemplo considerando los objetivos de desarrollo sostenible de Naciones Unidas), desarrolla en el estudiante el espíritu de iniciativa y creatividad, pero también ahonda en el sentido de la responsabilidad TURULL, (2020). Ahora bien, concentar en un proyecto los aprendizajes específicos de una asignatura no es una tarea fácil para desarrollar en las pocas semanas docentes de un cuatrimestre. Una vez más, para llevar a cabo este tipo de iniciativas, se requiere de la colaboración de los profesores que comparten docencia en un mismo cuatrimestre. Se deben buscar puntos de intersección y encontrar proyectos comunes que den sentido a los aprendizajes programados, para que constituyan una fuerte motivación para aprender, sin que se produzcan competencias entre las asignaturas y sin dilemas en el estudiante por si debe dedicar más tiempo a una u otra asignatura. Realizar proyectos reales no debe suponer una carga de trabajo inalcanzable para los estudiantes. La planificación de cada docente no debería ser exclusivamente individual, sino que es trascendental considerar que forma parte de un equipo docente. Cada profesor elabora su plan docente, tal vez para cada semana del curso, que implementará de forma independiente, pero deberá coordinarse con los otros profesores que imparten docencia en el mismo grupo de alumnos, para evitar semanas pico de entrega de trabajos.

\section{5.- Conclusiones}

La programación de las actividades en las distintas etapas del proceso de aprendizaje desarrolladas en la Clase Inversa online o presencial son muy similares. De este modo, cuando se mezclan espacios reales con entornos online, la Clase Inversa es fundamental para vertebrar la evolución de la docencia. Los entornos online y presenciales son fácilmente adaptables y se puede pasar de un entorno a otro con mucha facilidad. No obstante, las tutorías adquieren una especial relevancia en el entorno online.

La metodología desarrollada mediante la Clase Inversa permite alcanzar resultados de aprendizaje, y consecuentemente académicos, similares incluso mejores en un entorno online, si se consigue promover la responsabilidad y constancia del estudiante. Sin embargo, en este entorno se produce una polarización y se hace más evidente la falta de implicación de los estudiantes y sus malos resultados.

En el entorno online, la motivación extrínseca puede adquirir un papel relevante. En este sentido, el mejor camino para conseguir esta motivación es la coordinación de los profesores que imparten docencia en un mismo cuatrimestre, para diseñar proyectos comunes que consoliden los resultados de aprendizaje de cada asignatura.

La propuesta de mejora consistiría en plantear la metodología Clase Inversa y un aprendizaje basado en problemas y proyectos coordinado, para conseguir que los estudiantes puedan aplicar aquellos 
conocimientos que les permite enfrentarse a un mundo real y promueva su motivación por estudiar. En este contexto, la metodología de Clase inversa puede resultar idónea en cualquier ámbito, online o presencial.

\section{6.- Referencias}

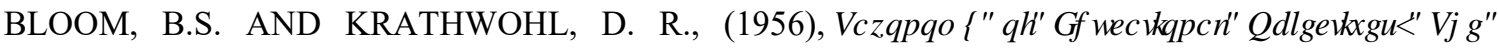

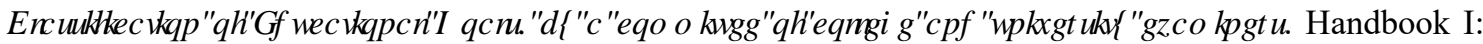
Cognitive Domain. NY, NY: Longmans, Green.

CASTILLA-CABANES, N., MARTÍNEZ-ANTÓN, A., TORMO-CLEMENTE, I., BLANCA-JIMÉNEZ,

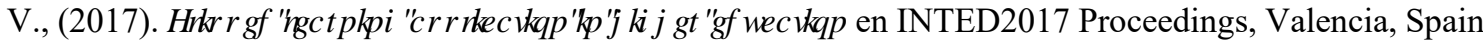
(pp. 6208-6217).

CASTILLO I VASQUEZ, (2003). ( OUJRUP HRRRQJIFRHQQDIQYHMW DFIYQFXDOMUMD. Revista Colombia Medica, 34 (3), 164-167.

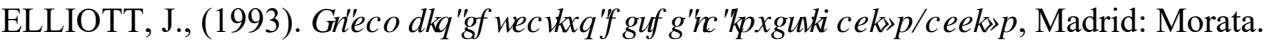

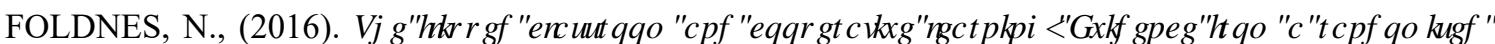

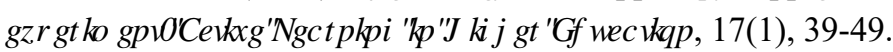

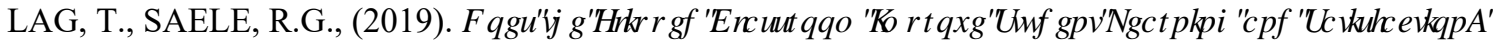

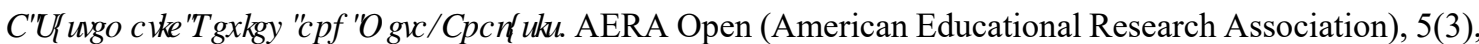
$1-17$.

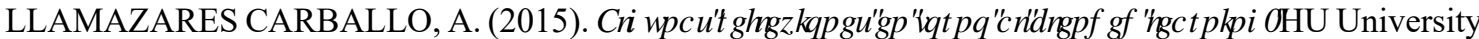
of Applied Sciences Utrecht. El Guiniguada. Revista de Investigaciones Y Experiencias En Ciencias de La Educación, 23(2014), 63-70 HU University of Applied Sciences Utrecht.

MAX TURULL, (2020). O DQXDO GH GRFHQID XQIYHWWDD. Colección Educación universitaria OCTAEDRO - IDP/ICE, UB

https://www.youtube.com/watch?v=2b3xG_YjgvI\#action=share.

MERLA GONZÁLEZ, A. E., (2016). El aula invertida como estrategia para la mejora del rendimiento académico. Revista Mexicana de Bachillerato a Distancia, 16(8), 67-77

MORALES SÁNCHEZ J.C., MARTÍNEZ NAHARRO S., CÁCERES GONZÁLEZ P., TURRO C. (2019). Flipped learning in an immature world. keys of methodology based on the Universitat Politècnica de València experience. 13th International Technology, Education and Development Conference. DOI:10.21125/inted.2019.0820

SALAS-RUEDA, R.A., Y LUGO-GARCÍA, J.L. (2019). Impacto del aula invertida durante el proceso educativo superior sobre las derivadas considerando la ciencia de datos y el aprendizaje automático. EDMETIC, Revista de Educación Mediática y TIC, 8(1), 147-170 doi:10.21071/edmetic.v8i1.9542

SOSA DÍAZ, M.J. Y PALAU MARTÍN, R.F. (2018) Flipped classroom para adquirir la competencia digital docente: una experiencia didáctica en la Educación Superior. Píxel-Bit. Revista de Medios y Educación, 52, 37-54 https://recyt.fecyt.es/index.php/pixel/article/view/62483.

doi:http://dx.doi.org/10.12795/pixelbit.2018.i52.03 


\section{7.- Agradecimientos}

La autora agradece la ayuda en la presentación de los datos objetivos realizada por H. Saenz de Juano Graduate Teaching Assistant in Economics. University of Glasgow. 